\title{
Voltage Contrast Imaging with Energy-Controlled Signal in an FE-SEM.
}

\author{
Y. Hashimoto ${ }^{1}$, S. Takeuchi ${ }^{1}$, T. Sunaoshi ${ }^{1}$, and Y. Yamazawa ${ }^{2}$ \\ 1. Application Development Dept., Hitachi High-Technologies Corporation, Kawasaki, Japan \\ 2. Electron Microscope Systems Design $1^{\text {st }}$ Dept., Hitachi High-Technologies Corporation, Hitachinaka, \\ Japan
}

Voltage contrast (VC) imaging with secondary electron (SE) signal in the scanning electron microscope (SEM) is basically used to visualize the difference of surface potential on local areas of the specimen. It is applied in the field of semiconductor industry such as failure analysis of devices [1]. The VC imaging can be also utilized to clarify compositional differences like the $\mathrm{Z}$ contrast imaging with backscattered electron (BSE), when the compositional differences of target materials make the differences of surface potential [2]. Even smaller compositional difference with which identification by $\mathrm{Z}$ contrast imaging is difficult, such as polymer and carbon, can be identified by the VC imaging in some cases [3]. However, the $\mathrm{VC}$ imaging method is not always easy, since it is easily affected by hydrocarbon contamination, and stabilizing status of charging balance. Therefore, it is important to optimize the observation and signal detection conditions for the VC imaging. In this study, optimization of these conditions such as incident energy, probe current of the SEM primary electron beam, and the detection conditions including energyfiltering conditions were examined.

Figure 1 (a) shows a general view of the Hitachi Regulus8220 FE-SEM. A cold field emission type electron source and a snorkel type objective lens which enables both high resolution imaging and flexibility of specimen size are adopted in the system. The beam deceleration function which allows ultralow voltage (ULV) imaging with high resolution capability is a standard feature of this model. Figure 1 (b) shows a schematic illustration of its signal detection system. The Regulus8220 has three types of SE detectors - top, upper, and lower Everhart-Thornley detectors - which provide valuable flexibility for imaging. The ratio of SE and BSE signals detected with the upper detector is controllable by using a signal control electrode installed inside the objective lens. Negative voltage applied to the signal control electrode suppress the low energy SE signal, and fine tuning of detected SE energy is possible by changing this suppression voltage.

Figure 2 shows an SE image of a Static Random Access Memory (SRAM) specimen. The specimen surface having many plugs connected to PMOS, NMOS and GATE is prepared by mechanical polishing. Three different plugs with different brightness are observed as indicated by circles (a), (b) and (c). The plug indicated by the circle (a) which is connected to PMOS appears the brightest, the plug indicated by the circle (b) which is connected to NMOS appears the second brightest, and the plug indicated by the circle (c) which is connected to GATE appears the darkest. The SE signal intensity at each plug varies with the surface potential made from the difference of inner structures such as pn junction and gate oxide of the plugs. This method is applicable for failure analyses of semiconductor devices. If there is a failure, abnormal contrast will appear at the failure position.

References:

[1] R. Rosenkranz, J. Mater. Sci.: Mater. Electron 22, 1523 (2011).

[2] S. Takeuchi et al., Microsc. Microanal. 16 (Suppl 2), 616 (2010).

[3] D. A. Dikin et al., Microsc. Microanal. 12 (Suppl 2), 674 (2006). 


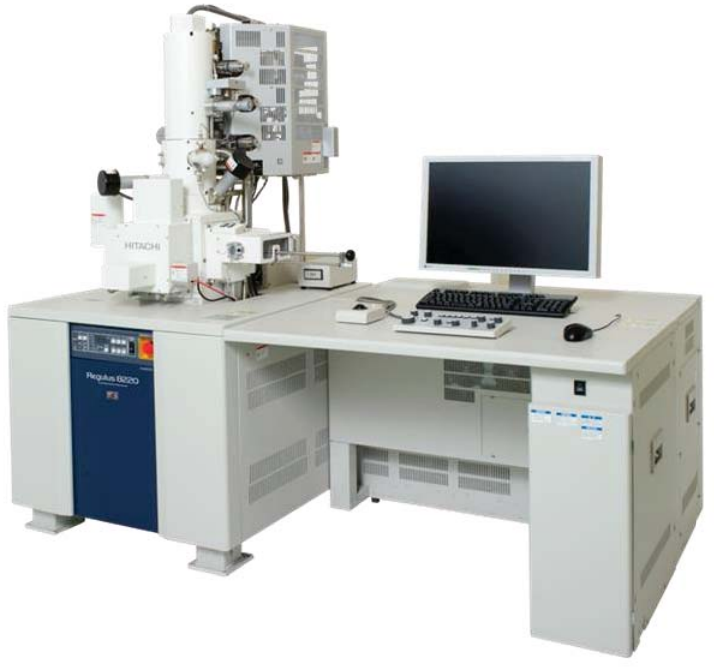

(a)

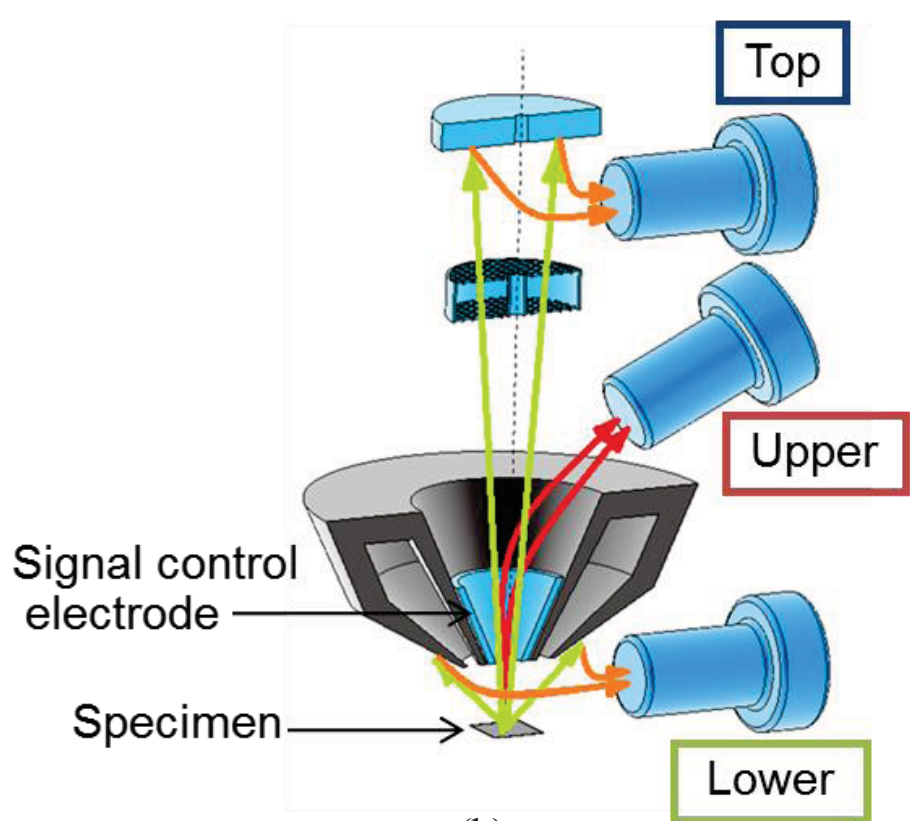

(b)

Figure 1. (a) General view of the Hitachi Regulus8220 FE-SEM and (b) Schematic illustration of signal detection system.
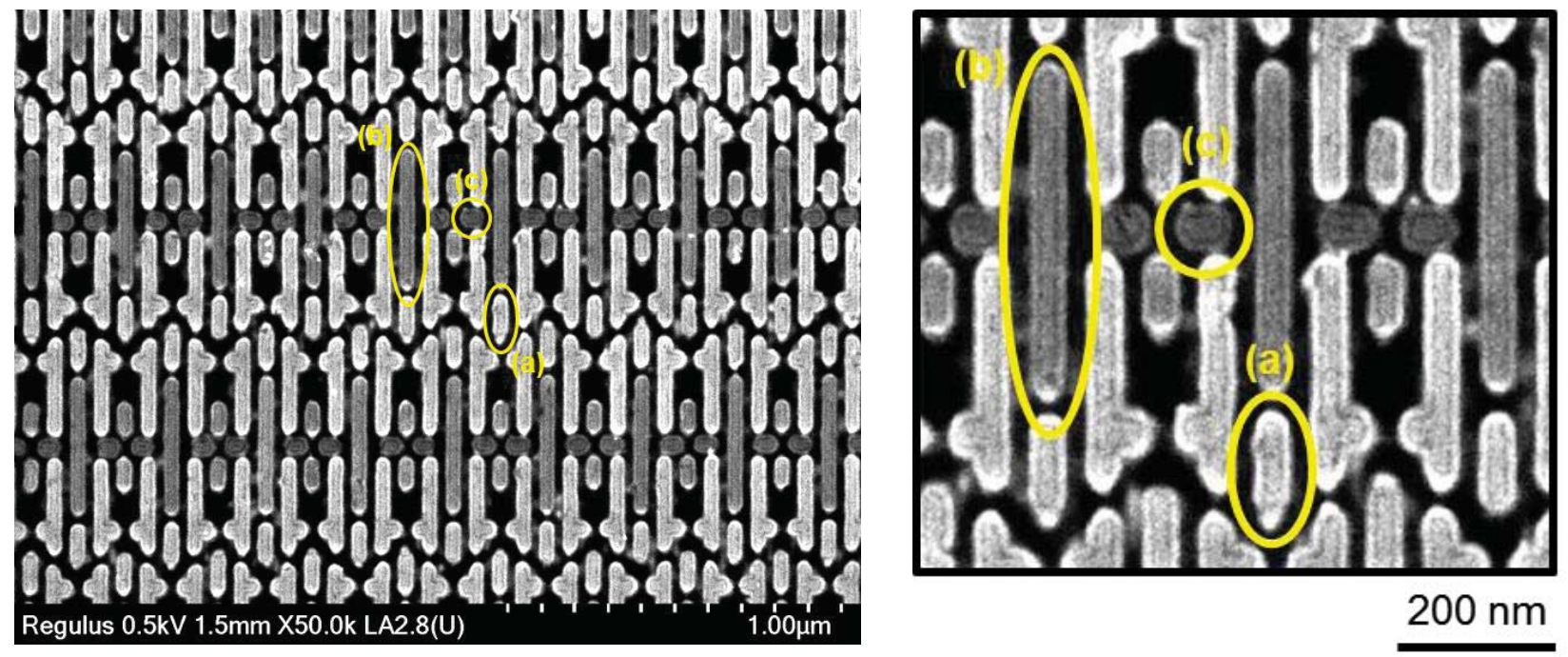

Figure 2. SE image of an SRAM specimen. Circles (a), (b) and (c) indicate PMOS, NMOS and GATE, respectively. 